\title{
Descripción de las Especies del Género Ipomoea Presentes en el Area de Riego del Río Dulce, Santiago del Estero, Argentina ${ }^{1}$
}

\author{
Description of the Species of the Genus Ipomoea in the Irrigated Area of Río Dulce, Santiago \\ del Estero, Argentina
}

CARRIZO, E. del V. ${ }^{2}$ y SOBRERO, Y.M.T. ${ }^{2}$

\begin{abstract}
RESUMEN - El objetivo del presente trabajo fue relevar y diferenciar especies del género Ipomoea presentes en diferentes fases de desarrollo, en el área de riego del Río Dulce, Santiago del Estero, Argentina. Para ello fueron herborizados y fotografiados diversos ejemplares encontrados en la región. En el laboratorio, después del trabajo de identificación, fueron descritas las diversas fases de desarrollo de la planta: semilla, plántula y estado adulto, creándose códigos de identificación para cada fase. Se registraron ocho especies del género Ipomoea, I. alba; I. amnicola; I. cairica; I. carnea; I. grandifolia; I. nil; I. purpurea e I. quamoclit, algunas de las cuales se cultivan como ornamentales, otras aparecen como ruderales y algunas son malezas en cultivos de la región.
\end{abstract}

Palabras Claves: malezas, ruderal, semilla, plántula, estado adulto.

ABSTRACT - The objective of this research was to describe and differentiate the stages of species of the genus Ipomoea in the irrigated area of Rio Dulce - Santiago del Estero - Argentina, under different growth stages. Species prototypes from the area were mounted in a herbarium, photographed and then identified with detailed descriptions of the seed, seedling and mature stages. A classification key was constructed to identify them under different growth stages. Eight species in the genus Ipomoea: (I. alba, I. amnicola, I. cairica, I. carnea, I. grandifolia, I. nil, I. purpurea and I. quamoclit) were registered, some cultivated as ornamental, while others appear naturally or as crop weeds in the region.

Key words: weeds, naturally, seed, seedling, mature stage.

\section{INTRODUCCIÓN}

El género Ipomoea comprende aproximadamente 500 especies, de las cuales alrededor de 200 se encuéntran en América tropical y subtropical. De éstas, 50 crecen en Argentina (O’Donell, 1959; Das et al., 1995). El género Ipomoea es el más numeroso y destacado de la familia Convolvulaceae por la importancia económica de muchas de sus especies, ya que algunas son utilizadas en la alimentación humana, otras como ornamentales y algunas han sido registradas como malezas, siendo en algunos casos tóxicas para el ganado.

En Santiago del Estero, en el área de riego del Río Dulce, es común encontrarlas como ruderales, como ornamentales o invadiendo cultivos.

El objetivo del presente trabajo fué relevar y diferenciar las especies de Ipomoea presentes en el área de riego del Río Dulce en las diversas fases de desarrollo: semilla, plántula y planta adulta.

\section{MATERIALES Y METODOS}

Este estudio fué realizado entre 1996 y 1998 durante el verano y el otoño, época en la que normalmente florecen y fructifican las especies de este género.

Los ejemplares se herborizaron y fotografiaron. Las flores y frutos se colocaron en una

Recebido para publicação em 16/3/2000 e na forma revisada em 26/1/2001.

2 Docentes, Facultad de Agronomía y Agroindustrias - UNSE. Avda. Belgrano (s) 1912. 4200 Santiago del Estero, Argentina. 
solución de formol, ácido acético glacial y alcohol. Se recolectaron semillas que fueron conservadas en bolsas de papel en condiciones de laboratorio y posteriormente fotografiadas con lupa.

En el laboratorio se procedió a la identificación de especies en estado adulto. Su descripción se realizó con datos obtenidos de las observaciones del material recogido y de la bibliografía: Burkart (1978); Cabrera (1983); Kissman y Groth (1992); Lorenzi (1994); Marzoca (1976); Parodi (1959).

Para el reconocimiento de plántulas se sembraron semillas de diferentes especies las cuales se mantuvieron en invernáculo hasta la aparición de 1 a 2 hojas verdaderas. Posteriormente se observaron características tales como: tamaño, forma, pilosidad de los cotiledones y el primer par de hojas verdaderas. Con este procedimiento fué posible diferenciar las especies en esta fase de desarrollo. Para la diferenciación de especies en fase de semilla se tuvo en cuenta color, pilosidad, forma y tamaño.
A partir de las descripciones se crearon códigos para identificar las especies en las fases de semilla, plántula y planta adulta.

\section{RESULTADOS}

Fueron registradas ocho especies del género Ipomoea: I. alba L.; I. amnicola Morong.; I. cairica (L.) Sweet.; I. carnea Jacq.; I. grandifolia (Dammer) O'Don.; I. nil (L.) Roth; I. purpurea (L.) Roth e I. quamoclit L.

De las especies registradas algunas se cultivan como ornamentales, tanto en las ciudades como en las áreas periféricas, I. alba; I. carnea; ; I. purpurea e I. quamoclit.

Aparecen como ruderales, cubriendo alambrados y vegetación, I. alba; I. amnicola; I. cairica e I. purpurea.

I. grandifolia; I. nil e I. purpurea son malezas en los cultivos de algodòn, soja y maíz. Seguidamente se presentan las claves para diferenciar las especies en las distintas fases de crecimiento.

\section{CLAVE DE SEMILLAS (Figura 1)}

A- Mayores de $8 \mathrm{~mm}$ de largo.

B- Negras, glabras excepto por diminutos pelos adpresos en el hilio.

BB- Pardas; pubescencia en toda la superficie; pelos lanosos y pardos, largos. I. alba (foto 1)

AA- Menores de $8 \mathrm{~mm}$ de largo.

B- Parduscas, glabras, lisas, levemente lustrosas. I. carnea (foto 2)

BB- Semillas sin tales caracteres.

C- Con pelos largos en los bordes.

D- Semillas pardoferrugíneas, con tomento denso, antrorso y pelos de los bordes seríceos, más claros. I. amnicola (foto 4)

DD- Semillas parduscas, de 5-6.5 mm de largo, con tomento fino y pelos lanosos, caducos, en los bordes. I. cairica (foto 5)

CC- Sin pelos en los bordes.

D- Semillas alargado-ovoides con pelos de diferente longitud que le dan un aspecto rugoso e irregularmente manchado de ceniza.

- Forma y pilosidad no sin tales caracteres.

E- Hilio con largos pelos adpresos y dirigidos al centro; dorso con surco profundo en el centro; caras ventrales planas a levemente cóncavas. I. nil (foto 7)

EE- Hilio glabro y áspero circundado por un anillo de diminutos pelos caedizos; dorso con surco mediano más o menos superficial; caras ventrales con una o dos arrugas transversales. I. purpurea (foto 8) 


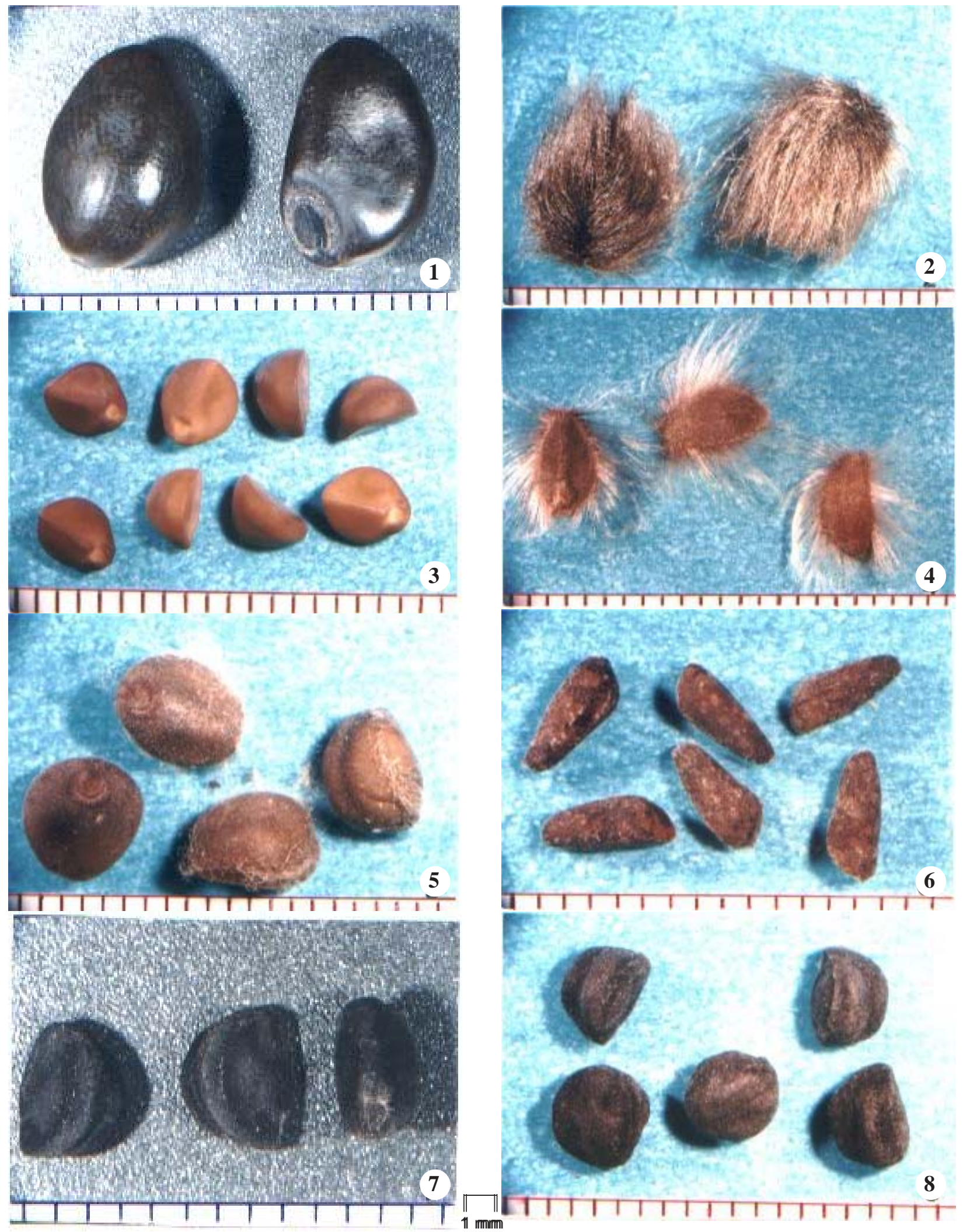

Figura 1 - Foto 1: I. alba; Foto 2: I. carnea; Foto 3: I. grandifolia; Foto 4: I. amnicola; Foto 5: I. cairica; Foto 6: I. quamoclit; Foto 7: I. nil; Foto 8: I. purpurea. 


\section{CLAVES DE PLÁNTULAS (Figura 2)}

A- Hojas cotiledonares con dos largos lobos estrechos,

divergentes, con ángulo de separación de generalmente $120-150^{\circ}$. Hojas definitivas pinatisectas. I.quamoclit (foto 3)

AA- Hojas cotiledonares sin tales caracteres.

B- Hojas cotiledonares con limbo muy grande de hasta

$5 \mathrm{~cm}$ de largo x $9 \mathrm{~cm}$ de ancho, con dos lobos

divergentes en un ángulo de $100^{\circ}$, anchos. I.alba (foto 1)

BB- Tamaño de las hojas cotiledonares sin tales caracteres.

C- Hojas definitivas 3-5-lobado-palmadas. I. cairica (foto 4)

CC- Hojas definitivas con limbo de base cordada y con tres lobos bien definidos, todos con ápice acuminado. Numerosos pelos en ambas caras. I.nil (foto 5)

CCC- Hojas definitivas cordiformes.

D- Hojas cotiledonares con dos anchos lobos, con puntos translúcidos en el envés que corresponden a glándulas. - I. purpurea (foto 8)

DD- Hojas cotiledonares sin tales caracteres.

E- Limbo de los cotiledones liso y glabro, con dos lobos redondeados proyectados lateralmente y dos lobos divergentes con ángulo de $60^{\circ}$, estrechos y con ápice obtuso. I. grandifolia (foto 6)

EE- Limbo sin tales caracteres.

F- Hojas cotiledonares de base truncada, lobos alargados y ápice redondeado, que divergen en un ángulo entre 45 y $70^{\circ}$. I. carnea (foto 2)

FF- Cotiledones sin tales caracteres. I.amnicola (foto 7 )

\section{CLAVES DE LAS ESPECIES EN ESTADO ADULTO (Figura 3)}

A- Arbusto erecto. I. carnea (foto 1)

AA- Plantas volubles.

B- Hojas palmatipartidas o pinatisectas.

C- Láminas 5-palmatipartidas, con segmentos lanceolados, elípticos u ovados, obtusos a agudos. I. cairica (foto 2)

CC- Láminas profundamente pinatisectas, con segmentos lineares, agudos.

BB- Hojas enteras

C- Enredadera robusta, perenne, con ramitas lisas o notablemente verrugosas. Sépalos exteriores con una arista carnosa. Corola hipocraterimorfa, de 7-13 cm de largo, blanca. I. alba (foto 4)

CC- Aspecto de la planta, ramas, sépalos y corola sin tales caracteres.

D- Dorso de los sépalos (especialmente la base) con largos pelos hirsutos, de base ensanchada.

E- Sépalos subiguales, terminados en largo acumen linear. Corola celeste o azul, más raro blanca, con el interior del tubo generalmente más pálido.

EE- Sépalos agudos a acuminados (no largamente). Corola azul, rosada o blanca, a veces variegada. __

DD- Sépalos exteriores glabros o apenas pilosos, raro muricados en el dorso.

E- Cápsulas ovoideas, glabras, terminadas en un apículo caduco, acompañadas por los sépalos reflexos. — I. amnicola (foto 7)

EE- Cápsulas subglobosas, algo deprimidas, hirsuto-pilosas.

I. grandifolia (foto 8) 

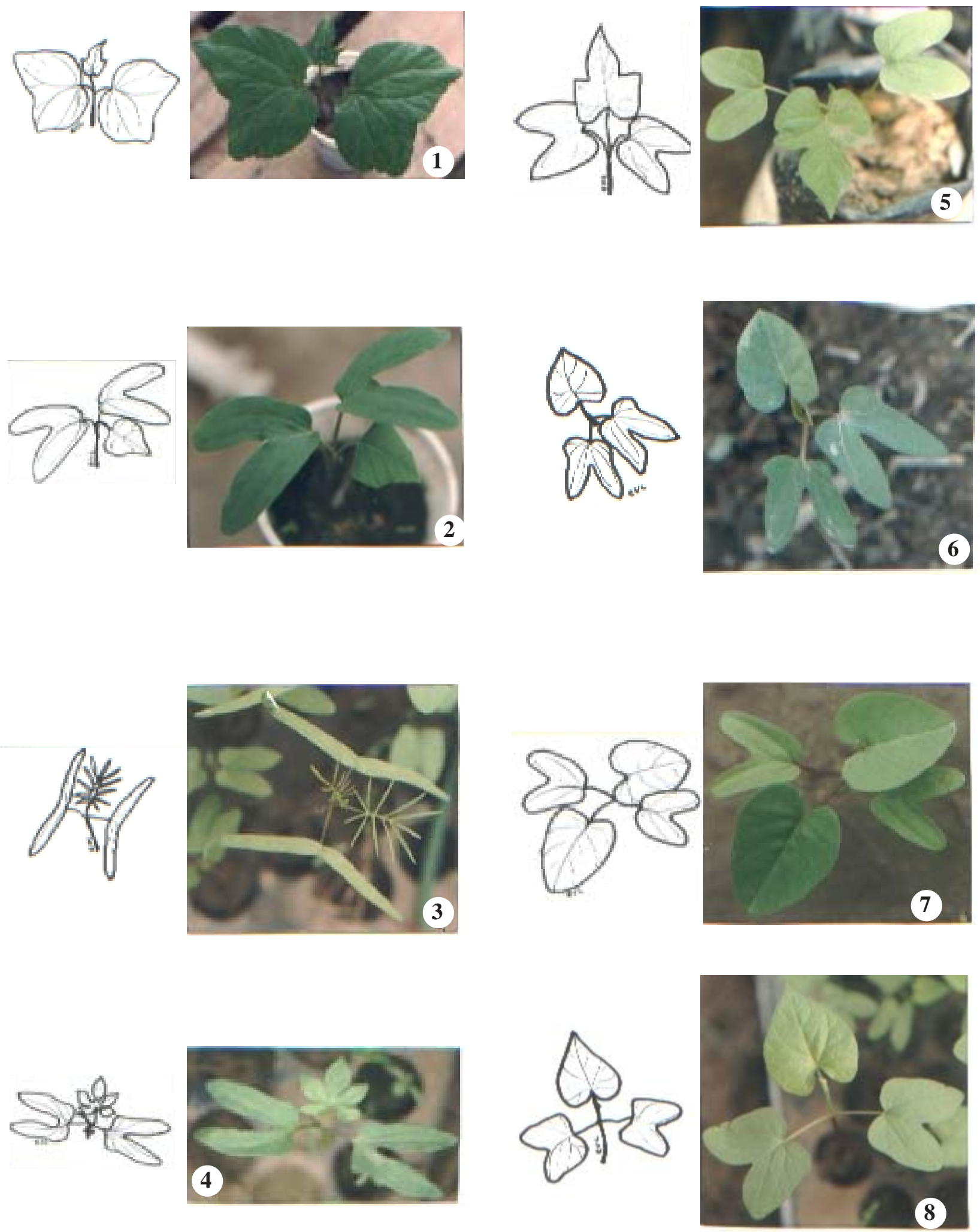

Figura 2 - Foto 1: I. alba; Foto 2: I. carnea; Foto 3: I. quamoclit; Foto 4: I. cairica; Foto 5: I. nil; Foto 6: I. grandifolia; Foto 7: I. amnicola; Foto 8: I. purpurea. 

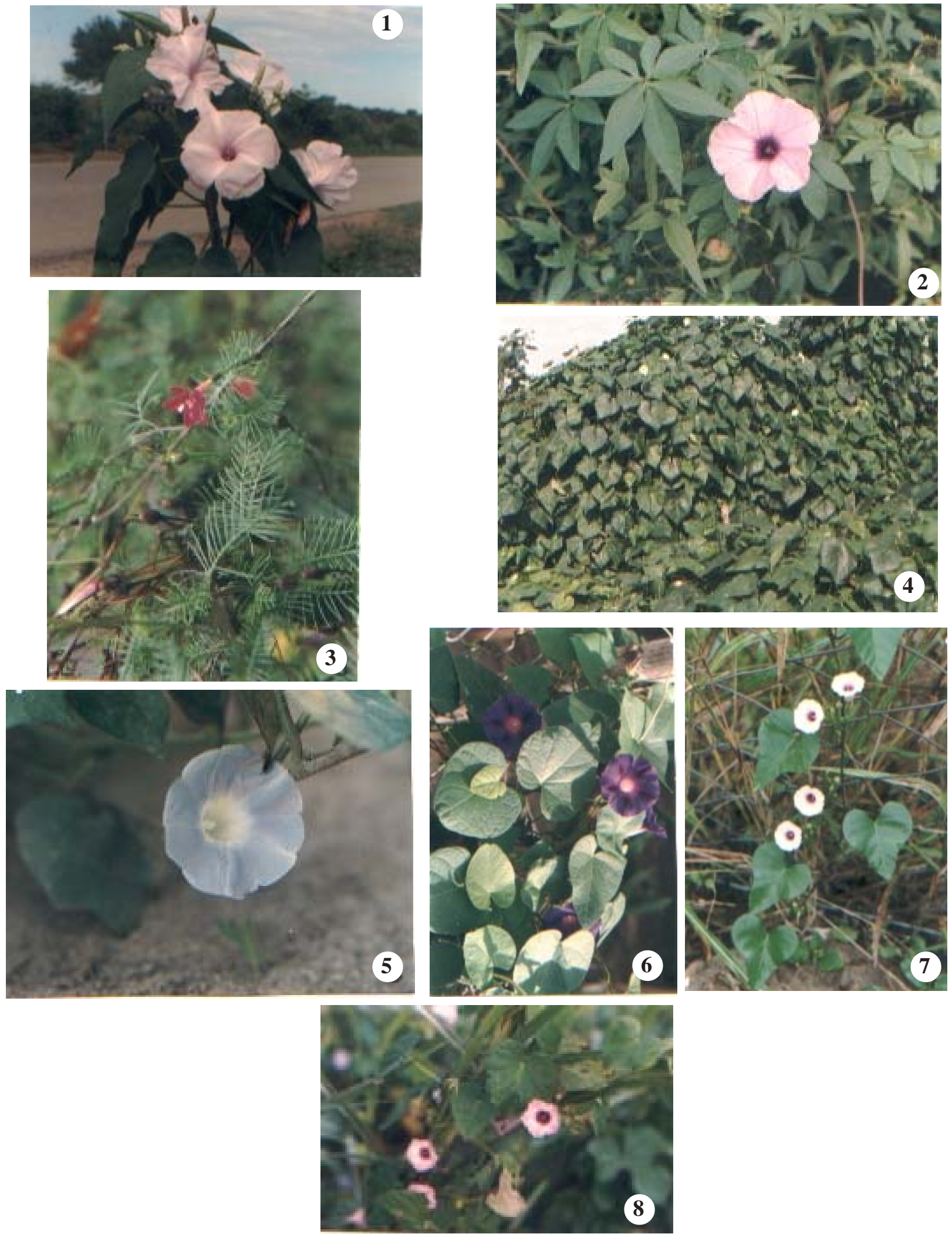

Figura 3 - Foto 1: I. carnea; Foto 2: I. cairica; Foto 3: I. quamoclit; Foto 4: I. alba; Foto 5: I. nil; Foto 6: I. purpurea; Foto 7: I. amnicola; Foto 8: I. grandifolia. 


\section{AGRADECIMIENTOS}

Los autores expresan su agradecimiento al Ing. Agr. Salvador Chaila, Dr. Roberto Manes y Licenciado Fernando Campos por las fotografias de semillas de esta publicación.

\section{LITERATURA CITADA}

BURKART, A. Flora ilustrada de Entre Rios. Col. Cient. INTA., v.6, n.5, p.1-606, 1978. (Primulales-Plantaginales)

CABRERA, A.L. Flora de la Provincia de Jujuy. Col. Cient. INTA. v.8, n.8, p.1-508 1983. (Clethraceas a Solanáceas)

DAS, S., MITRA, S.K., MUKHERJEE, K.K. Sem studies on seed coat of some species of Ipomoea (Convolvulaceae). Phytomorphology, v.45, n.1/ 2, p.113-125, 1995.
KISSMANN, K.G., GROTH,D. Plantas infestantes e nocivas. Tomo 2: dicotiledôneas. São Paulo: BASF Brasileira S.A. 798p. 1992.

LORENZI, H. Manual de identificação e controle de plantas daninhas. 4.ed. Nova Odessa: Plantarum, 1994.

MARZOCCA, A. Manual de malezas, 4.ed. Buenos Aires: Hemisferio Sur, 684p. 1976.

O’DONELL, C.A. Las especies americanas de Ipomoea L. Sect. Quamoclit (Moench) Griseb. Lilloa v.29, p.19-86, 1959.

PARODI, L.R. Enciclopedia Argentina de Agricultura y Jardineria. 2.ed. Buenos Aires, v.1, p.25-47, 1959. 